\title{
Erratum: Testing Nonassociative Quantum Mechanics [Phys. Rev. Lett. 115, 220402 (2015)]
}

\author{
Martin Bojowald, Suddhasattwa Brahma, and Umut Büyükçam \\ (Received 2 August 2016; published 22 August 2016)
}

DOI: 10.1103/PhysRevLett.117.099901

This Letter corrects a sign mistake made in solving an uncertainty relation for minimal momentum fluctuations, and discusses implications for the resulting physical effect. In the Letter, we first solved semiclassical equations of motion for stationary second-order moments, and then imposed minimal uncertainty by saturating uncertainty relations. This led us to the equations $\Delta\left(p_{x}^{2}\right)=\Delta\left(p_{y}^{2}\right), \Delta\left(p_{x} p_{y}\right)=0$ and

$$
\Delta\left(p_{x}^{2}\right) \Delta\left(p_{y}^{2}\right)-\Delta\left(p_{x} p_{y}\right)^{2} \geq \frac{1}{4} e^{2} \hbar^{2}\langle\hat{B}\rangle^{2}
$$

which we saturated by $\Delta\left(p_{x}^{2}\right)=\frac{1}{2} e \hbar\langle\hat{B}\rangle$. However, the magnetic field can be negative, and therefore we should have written $\Delta\left(p_{x}^{2}\right)=\frac{1}{2} e \hbar|\langle\hat{B}\rangle|$ (assuming $e>0$ ). These fluctuations enter the effective potential which now reads

$$
V_{\mathrm{eff}}(\langle\hat{z}\rangle)=\frac{1}{2} m \omega^{2}\langle\hat{z}\rangle^{2}+\frac{1}{2} \hbar \frac{e|B(\langle\hat{z}\rangle)|}{m}+\frac{1}{2} \hbar \omega .
$$

In our specific example, $B(\langle\hat{z}\rangle)=\mu\langle\hat{z}\rangle$ appeared to lead to a shift of the minimum of the effective potential by quantum effects. However, with the absolute value in (2), the effective potential is still reflection symmetric around $\langle\hat{z}\rangle=0$, and there is no shift of the minimum. Instead, the effective potential differs from the classical potential by a kink around $\langle\hat{z}\rangle=0$, accompanied by a modification of the potential in a neighborhood around $\langle\hat{z}\rangle=0$ where the linear contribution to the potential is dominant. (At the kink, where the $\hbar$ correction implied by the magnetic field is zero, it is likely that higher-order corrections are relevant.) The motion of a charged particle in a magnetic monopole density therefore differs from the classical motion by anharmonic behavior around the minimum of an additional quadratic potential.

In summary, our main result of giving a physical interpretation to nonassociative quantum mechanics and providing new methods to make effects calculable remains unchanged. However, in terms of potential observations in the specific example provided by us, this implies that one would have to detect deviations from harmonic motion rather than a shift of the equilibrium position, as reported earlier. 